\title{
An asymmetric dimer in a periodic potential: a minimal model for friction of graphene flakes
}

\author{
Remco Hens and Annalisa Fasolino ${ }^{\mathrm{a}}$ \\ Radboud University Nijmegen, Institute for Molecules and Materials, Heyendaalseweg 135, 6525 AJ Nijmegen, The Netherlands
}

Received 4 May 2016

Published online 11 July 2016

(c) The Author(s) 2016. This article is published with open access at Springerlink.com

\begin{abstract}
We discuss the friction and motion of a model of a dimer with asymmetric interactions with a substrate potential. Starting from the consideration that a rigid dimer with spacing equal to half of the period of the potential has exactly zero static friction like the infinite incommensurate Frenkel Kontorova model, we show how stick-slip behaviour and friction arise as a function of asymmetry. We argue that this model can yield a simple yet insightful description of the frictional behaviour of graphene flakes on graphite and of superlubricity. The results can also be of interest for diatomic molecules on surfaces.
\end{abstract}

\section{Introduction}

The friction of crystalline layers sliding on incommensurate substrates can be extremely low [1,2], a phenomenon called structural superlubricity [3]. For nanometer sized graphene flakes sliding on graphite, the friction as a function of rotation angle between the flake and substrate displayed peaks for the perfect commensurate case (flake and substrate with the same orientation) and decreased rapidly for incommensurate cases [2]. In the last years, superlubricity is attracting increasing interest in the literature [4-7].

Superlubricity can be easily justified by means of the one-dimensional (1D) Frenkel Kontorova (FK) model describing a harmonic chain on a periodic potential. For incommensurate ratios of the chain lattice spacing $a$ to the period of the potential $b$ this model displays the so-called Aubry transition to a state of vanishing static friction for weak coupling to the substrate $[8,9]$. For incommensurate contacts, in fact, the total energy does not vary with the position of the chain onto the potential, formally corresponding to a continuous modulation function. This exact result applies in the thermodynamic limit of infinite chains. For a finite system, the difference between commensurate and incommensurate contacts is lost due to relaxation at the edges [10]. Therefore the fact that superlubricity has been observed for finite graphene flakes at incommensurate contact suggests that the relaxation at the edges is negligible. Indeed the graphitic bond is one of the strongest in nature and molecular dynamics simulations show that the bond length at the edges of graphene

\footnotetext{
* Supplementary material in the form of three mp4 files available from the Journal web page at

http://dx.doi.org/10.1140/epjb/e2016-70273-5

a e-mail: a.fasolino@science.ru.nl
}

flakes varies during the motion by less than $0.005 \AA$ with respect to the bulk value, preserving the incommensurate contact.

In experiments and simulations, the superlubric sliding is usually lost due to irreversible rotations to a commensurate contact $[4,11]$ with resulting high friction and stick-slip behaviour. However, it has been recently experimentally shown that friction can be increased reversibly by applying load to the incommensurate flake. This reversible transition from superlubric to stick-slip sliding occurs while the flake remains at incommensurate contact and can be attributed to vertical motion of the carbon atoms at the edge with negligible in-plane stretching, a mechanism not described by the standard 1D FK model. In the stick-slip regime, the friction increases with load but remains at least one order of magnitude smaller than for commensurate contact [12].

Here we propose a minimal model based on a dimer with asymmetric interactions with a periodic potential that well describes the behaviour observed for incommensurate graphene flakes under load [12]. The two particles in the dimer are meant to represent the edge and the centre of a flake with different interactions to the substrate. At the same time this model can be an interesting model to describe the motion of diatomic molecules on surfaces. Models of dimer in a symmetric potential under the effect of an external modulation breaking the symmetry and leading to ratchet effects have been previously studied $[13,14]$. The interest of the model we propose is that the limiting case of a symmetric, rigid dimer with interatomic distance equal to one half of the substrate periodicity has, like the infinite incommensurate FK model, the property that the energy does not depend on the position. In this respect, it represents a perfectly incommensurate finite system with vanishing static friction. This property makes it possible 


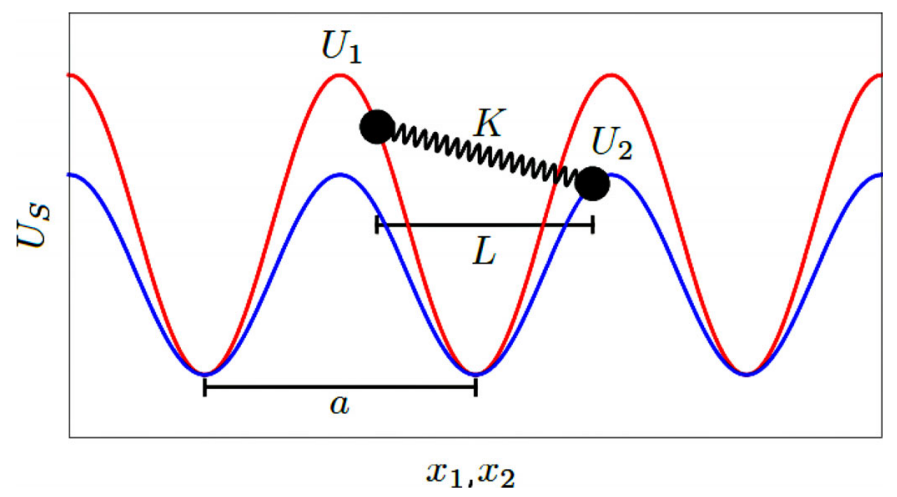

Fig. 1. The asymmetric dimer in a periodic potential.

to examine in general terms the effect of asymmetric interactions and deviations from perfect incommensurability on friction.

\section{Model}

We model a graphene flake by a 1D harmonic dimer formed by two particles with coordinates $x_{1}$ and $x_{2}$ connected by a spring of spring constant $K$ and rest length $L$. Particle 1 will represent the edge of the graphene flake and particle 2 the center of the flake. The harmonic potential energy is:

$$
U_{H}=\frac{K}{2}\left(x_{2}-x_{1}-L\right)^{2} .
$$

The graphite substrate is modeled by a periodic sinusoidal potential:

$$
U_{S}=U_{0}-U_{1} \cos \left(\frac{2 \pi x_{1}}{a}\right)-U_{2} \cos \left(\frac{2 \pi x_{2}}{a}\right)
$$

where $U_{1}$ and $U_{2}$ are the amplitudes of the sinusoidal potentials with periodicity $a$ and $U_{0}=U_{1}+U_{2}$. Figure 1 illustrates this model. By changing the values of $U_{1}$ and $U_{2}$ we change the behavior of edge and centre of the flake as will be explained later. By changing the ratio $L / a$ the commensurability of the system is changed. A ratio $L / a=1$ gives a commensurate contact. An incommensurate contact is realized by a rigid dimer $(K \rightarrow \infty)$ with $L / a=1 / 2$. In fact, if $U_{1}=U_{2}$, the total energy $U_{H}+U_{S}$ does not depend on the position of the dimer on the substrate as for the incommensurate FK model. One atom can be at the bottom and one at the top of $U_{S}$ or at any intermediate position without energy change, implying a vanishing friction force (superlubricity) to displace the dimer on the substrate. We note that $1 / 2$ is also the first term in the Fibonacci series that approximates the irrational number $1 / \tau$ with $\tau=\frac{1+\sqrt{5}}{2}$, the golden mean for which the FK model has been much studied.

The sliding friction of the system, can be studied by means of the Tomlinson model [15]. The dimer is pulled at constant speed $v_{p}$ by a spring, with spring constant $K_{p}$, connected to the centre of mass of the dimer. The friction is defined as the time average of the spring force $F_{p}$ defined below.

Assuming the same mass for the two particles, changing to adimensional units (as in [16]):

$$
t \rightarrow \frac{2 \pi}{a \sqrt{m}} t, x_{i} \rightarrow \frac{a}{2 \pi} x_{i}, L \rightarrow \frac{a}{2 \pi} L, K \rightarrow \frac{4 \pi^{2}}{a^{2}} K
$$

and defining the center of mass and relative coordinate as:

$$
\left\{\begin{array}{l}
x_{C M}=\frac{1}{2}\left(x_{1}+x_{2}\right) \\
x_{R}=x_{2}-x_{1}-L
\end{array}\right.
$$

the equation of motion becomes:

$$
\left\{\begin{aligned}
\ddot{x}_{C M}= & -\frac{1}{2} U_{+} \sin x_{C M} \cos \left(\frac{x_{R}+L}{2}\right) \\
& +\frac{1}{2} U_{-} \cos x_{C M} \sin \left(\frac{x_{R}+L}{2}\right) \\
& +K_{p}\left[v_{p} t-x_{C M}+x_{C M}(0)\right]-\eta \dot{x}_{C M} \\
\ddot{x}_{R}=- & 2 K x_{R}-U_{+} \cos x_{C M} \sin \left(\frac{x_{R}+L}{2}\right) \\
+ & U_{-} \sin x_{C M} \cos \left(\frac{x_{R}+L}{2}\right)
\end{aligned}\right.
$$

where we defined $U_{+}=U_{1}+U_{2}$ and $U_{-}=U_{1}-U_{2}$ and $\eta$ is a damping constant. In the Tomlinson model, the friction is given by the time average of the force in the pulling spring:

$$
F_{\text {friction }}=\frac{1}{T} \int_{0}^{T} F_{p} d t
$$

where $T$ is the period of the motion of the dimer and

$$
F_{p}=K_{p}\left[x_{C M}(0)+v_{p} t-x_{C M}(t)\right] .
$$

We solve the equation of motion by the Runge Kutta 2 (or improved Euler) method with time step (in adimensional units) $h=10^{-4}$ giving conservation of energy. Furthermore $\eta=2, K_{p}=0.4$ and $v_{p}=10^{-3}$ are used.

\section{Results}

We first consider a symmetric dimer $\left(U_{1}=U_{2}\right)$. In Figure 2 we compare the behavior of $F_{p}$ and $x_{C M}$ as a function of time for an incommensurate dimer $(L / a=1 / 2)$ with different values of $K$. A rigid dimer has $K=\infty$, namely a fixed length. The motion of a rigid dimer follows the pulling spring without any stretching whereas, for decreasing spring constant, the motion becomes more of stick-slip type resulting in higher friction. The case of a rigid dimer, describes well a graphene flake where the in-plane distortions are negligible.

In Figure $3 F_{\text {friction }}$ is shown as a function of $L$ for the symmetric periodic potential $U_{1}=U_{2}$. For weakly bound dimers (with small $K$ ) the friction is (almost) independent of $L$. In fact, the energy stored in the spring is small compared to substrate energy and therefore $L$ does not play a large role. For more strongly bound dimers $(K>0.20)$, the friction has always a minimum at $L / a=1 / 2$, corresponding to the incommensurate case, and maxima at 

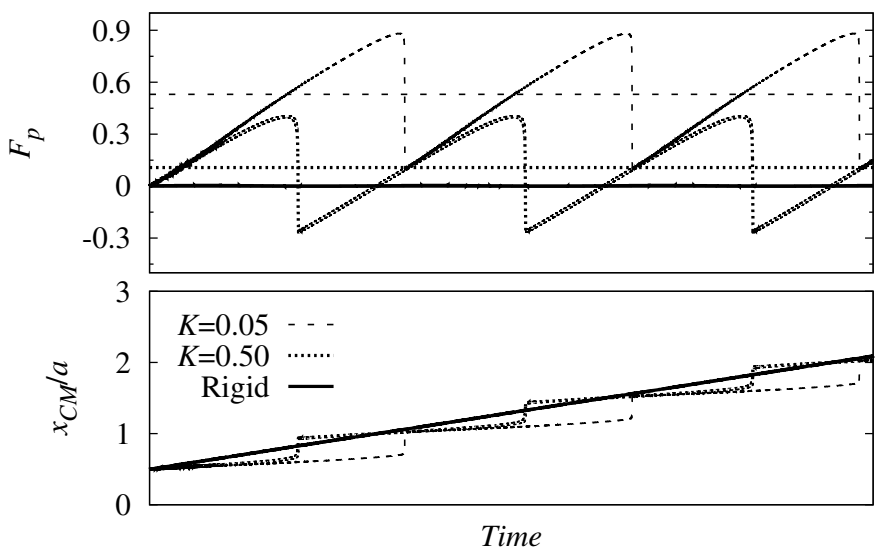

Fig. 2. Force in the pulling spring, $F_{p}$, and position of the centre of mass as a function of time for a dimer with $L / a=1 / 2$ and different values of $K$ in a potential with $U_{1}=U_{2}=1.00$. The friction, $F_{\text {friction }}$ is, the average spring force (Eq. (6)) (horizontal lines). The rigid dimer has zero friction and a continuous motion. With decreasing spring constant, the friction increases and the motion evolves to a stick-slip behaviour.

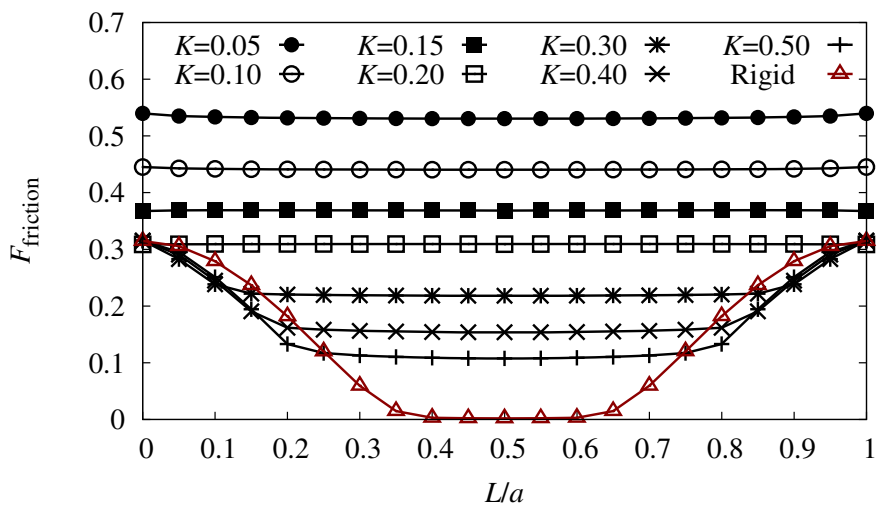

Fig. 3. Friction of the dimer as a function of its rest length $L$ for the symmetric potential: $U_{1}=U_{2}=1.00$ and different values of $K$.

$L / a=0$ and $L / a=1$, corresponding to the commensurate case. A comparison for this case of Figure 3 with Figure 3 of reference [2] shows a qualitative agreement with the experimental results for graphene flakes on graphite. The friction minima and maxima can be understood by looking at the motion of the dimer as shown in Figure 4 for the rigid dimer with $L / a=1 / 2$ and $L / a=1$.

These results for symmetric dimers show that the onset of friction is related to relaxation of the dimer in the periodic potential, leading to variation of the interatomic distance $x_{2}-x_{1}$. As already discussed, the strong bonding in graphene does not lead to substantial in-plane relaxation at the edges that can explain the experimentally observed transition from continuous to stick-slip motion. We show now, that asymmetry of the interactions with the substrate $\left(U_{1} \neq U_{2}\right)$ can lead to stick-slip behaviour even for a rigid dimer, with consequent friction, albeit much lower than for commensurate cases.

Figure 5 shows the friction as a function of $L$ but this time for $U_{1}>U_{2}$. For the weakly bound dimers no
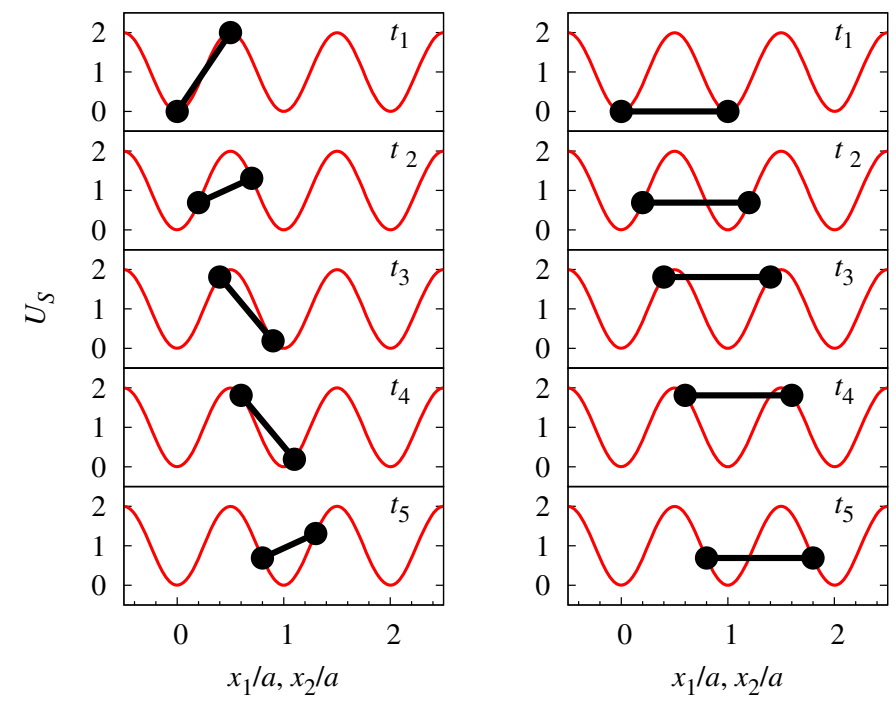

Fig. 4. Comparison of the motion of the incommensurate (left panel) and commensurate (right panel) rigid dimer in the symmetric potential with $U_{1}=U_{2}=1.00$. The positions of the dimer are shown at five successive, equally spaced, times. For $L / a=1 / 2$, motion increasing the potential energy of one particle is always compensated by the energy gain of the other. This conservation of energy results in zero friction. For the commensurate case $L / a=1$, both particles always gain or lose energy simultaneously resulting in maximal energy variations and the highest friction.

large differences are observed, the friction remains (almost) independent of $L$. For the more strongly bound dimers the minima and maxima have shifted (compared to Fig. 3). The most important, although less apparent, difference is that an asymmetric incommensurate rigid dimer $(L / a=1 / 2)$ has a small but non zero friction as we will discuss next. For completeness, we notice that for all other values of $K>0.20$ the minimum is located at a smaller value than $L / a=1 / 2$ and the maximum is at a value smaller than $L / a=1$. This shift can be explained again by considering the motion of the dimer. For simplicity we consider the dimer being pulled to the right with particle 2 in front of particle $1 x_{2}>x_{1}$. Since $U_{2}<U_{1}$ particle 2 is less bound by the substrate than particle 1 causing particle 2 to move to the right and particle 1 to lag behind, stretching the spring. A spring with shorter rest length reduces this effect so that the minimum friction shifts to a smaller value of $L$. From Figures 3 and 5 one can see that a rigid dimer does not always have the lowest friction: for $L / a$ close to 0 or 1 the lowest friction is achieved by strongly bound but not rigid dimers. This effect is stronger for the asymmetric case. The possible relative motion of the two particles allows the transformation of substrate potential energy $U_{S}$ in harmonic spring energy $U_{H}$ reducing the force on the pulling spring and thereby reducing the friction.

The model of a dimer in an asymmetric potential captures the effect of the flake edges on the friction. In [12], it was shown that, under load, the atoms at the edges of incommensurate graphene flakes have a larger 


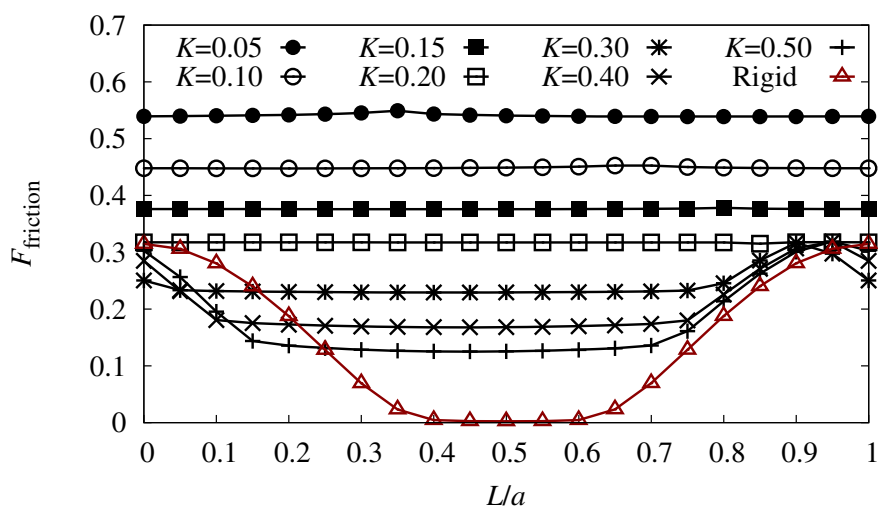

Fig. 5. Friction of the dimer as a function of its rest length $L$ for an asymmetric potential $U_{1}=1.20$ and $U_{2}=0.80$ and different values of $K$. The rigid dimer has $K=\infty$. For a rigid dimer with $L / a=0.5, F_{\text {friction }}$ is small but finite (see text).

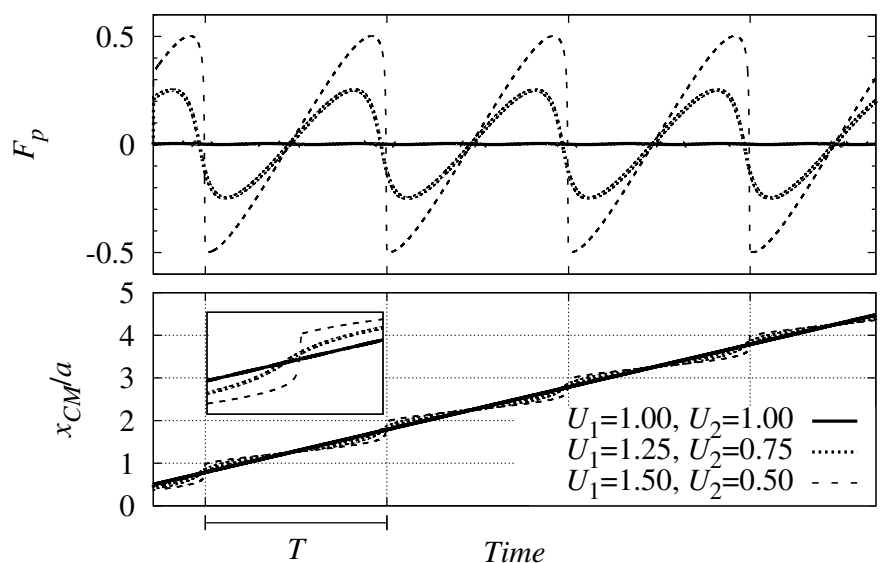

Fig. 6. Transition from continuous to stick-slip motion for the rigid dimer and $L / a=0.5$. The upper panel shows the spring force for three different values of $U_{1}$ and $U_{2}$ as a function of time and the lower panel shows the center of mass of the dimer. The inset is a zoom-in on the motion to show the difference between continuous and stick-slip motion. For $U_{1}=U_{2}=1.00$ the dimer moves continuously yielding (almost) zero friction. For $U_{1}=1.25$ and $U_{2}=0.75$ the motion evolves to stick-slip and the friction grows to $F_{\text {friction }}=2.6 \times 10^{-3}$. For $U_{1}=1.50$ and $U_{2}=0.50$ the motion is clearly stick-slip and $F_{\text {friction }}=$ $2.8 \times 10^{-2}$ (see also Fig. 7).

out-of-plane displacement than the central atoms. Since particle 1 is meant to describe the edge of the flake, we implement this effect by choosing a rigid incommensurate dimer with $U_{1}>U_{2}$. The effect of the asymmetry on the motion and friction is shown in Figure 6. With increasing asymmetry the motion goes from continuous to stick-slip and the force in the spring becomes more sawtooth like, with a small but non zero friction. In Figure 7 details of the motion are illustrated (see also the animations in the supplementary material). This change of behaviour with asymmetry is only observed for the rigid or strongly bound dimers, supporting this minimal model as representative of the behaviour of incommensurate graphene flakes under load.
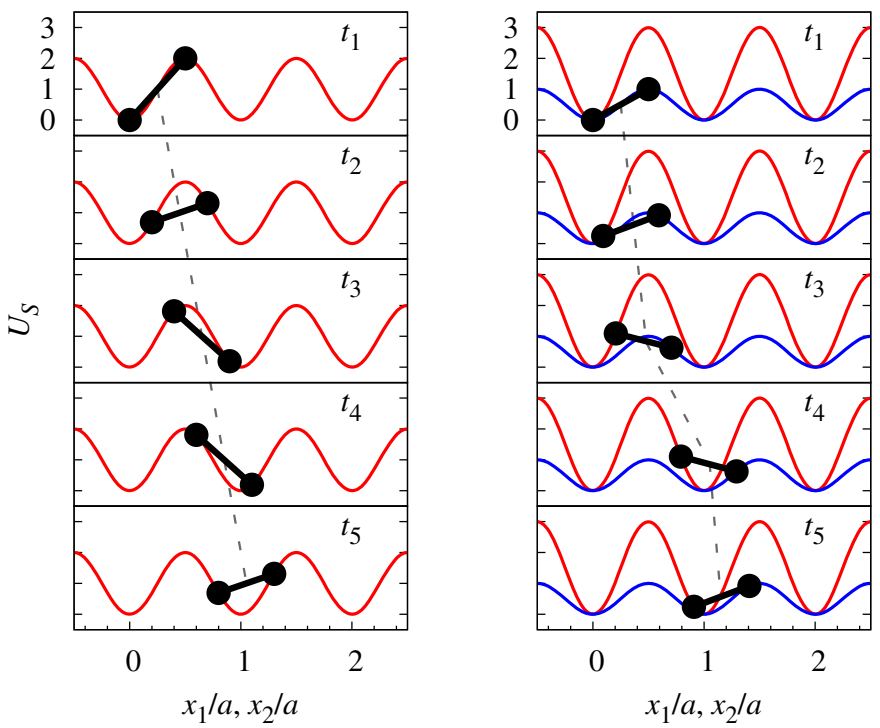

Fig. 7. Motion of the rigid dimer with $L / a=0.5$. The positions of the dimer are shown at five successive, equally spaced, times. The grey dashed line shows the position of the center of mass. The left panels show the motion for $U_{1}=U_{2}=1.00$, the center of mass moves in a straight line: the motion is continuous. The right panels show the motion for $U_{1}=1.50$ and $U_{2}=0.50$, now the center of mass makes a jump between $t_{3}$ and $t_{4}$ : the motion is stick-slip.

\section{Conclusion}

We have discussed the motion and friction of asymmetric dimers on a periodic potential and proposed this model as a minimal description of the behaviour and friction of incommensurate graphene flakes on graphite under load. The asymmetry of the interactions of the two atoms with the substrate is meant to describe the difference between edge and central atoms of graphene flakes. The results obtained for this model, give a simple qualitative description of the behaviour of graphene graphene flakes $[2,12]$ that can be insightful also for other situations in surface science.

We are very grateful to Merel van Wijk for many useful suggestions. A.F. acknowledges support of the Foundation for Fundamental Research on Matter (FOM), which is part of the Netherlands Organisation for Scientific Research (NWO) within the program n.129 "Fundamental Aspects of Friction". This work is supported in part by COST Action MP1303.

\section{References}

1. M. Hirano, K. Shinjo, R. Kaneko, Y. Murata, Phys. Rev. Lett. 78, 1448 (1997)

2. M. Dienwiebel, G.S. Verhoeven, N. Pradeepa, J.W.M. Frenken, J.A. Heimberg, H.W. Zandbergen, Phys. Rev. Lett. 92, 126101 (2004)

3. K. Shinjo, M. Hirano, Surf. Sci. 283, 473 (1993)

4. A.E. Filippov, M. Dienwiebel, J.W.M. Frenken, J. Klafter, M. Urbakh, Phys. Rev. Lett. 100, 046102 (2008) 
5. Z. Liu, J. Yang, F. Grey, J.Z. Liu, Y. Liu, Y. Wang, Y. Yang, Y. Cheng, Q. Zheng, Phys. Rev. Lett. 108, $205503(2012)$

6. Y. Liu, F. Grey, Q. Zheng, Sci. Rep. 4, 046102 (2014)

7. D. Berman, A. Erdemir, A.V. Sumant, Mater. Today 17, $31(2014)$

8. S. Aubry, Physica D 7, 240 (1983)

9. S. Aubry, P.Y. Le Daeron, Physica D 8, 381 (1983)

10. O.M. Braun, M. Peyrard, V. Bortolani, A. Franchini, A. Vanossi, Phys. Rev. E 72, 056116 (2005)

11. M.M. van Wijk, A.S. de Wijn, A. Fasolino, J. Phys.: Condens. Matter 28, 134007 (2016)

12. M.M. van Wijk, M. Dienwiebel, J.W.M. Frenken, A. Fasolino, Phys. Rev. B 88, 235423 (2013)

13. S. von Gehlen, M. Evstigneev, P. Reimann, Phys. Rev. E 79, $031114(2009)$
14. D. Speer, R. Eichhorn, M. Evstigneev, P. Reimann, Phys. Rev. E 85, 061132 (2012)

15. A. Fasolino, in Handbook of Theoretical and Computational Nanotechnology, edited by M. Rieth, W. Schommers (American Scientific Publishers, 2006)

16. C. Fusco, A. Fasolino, T. Janssen, Eur. Phys. J. B 31, 95 (2003)

Open Access This is an open access article distributed under the terms of the Creative Commons Attribution License (http://creativecommons.org/licenses/by/4.0), which permits unrestricted use, distribution, and reproduction in any medium, provided the original work is properly cited. 